\title{
Evaluation of Teaching Quality of College Physical Education Based on Analytic Hierarchy Process
}

\author{
$\underline{\text { https://doi.org/10.3991/ijet.v15i10.14039 }}$ \\ Ke Han \\ Harbin Institute of Technology (Weihai), Weihai, China \\ hankejiayoul 163. com
}

\begin{abstract}
There are many difficulties in the evaluation of the teaching quality of physical education (PE) in colleges: the evaluation factors are complicated, the evaluation systems are incomplete, and the evaluation methods are not comprehensive enough. To overcome these difficulties, this paper introduces analytic hierarchy process (AHP) to the evaluation of PE teaching quality in colleges. Firstly, the authors identified the influencing factors of PE teaching in colleges today. Next, an index system was established for the evaluation, and an evaluation model was set up based on the AHP and grey system theory (GST). Finally, our method was proved feasible through example analysis. The research results provide new insights on the application of state-of-the-art theories in quality evaluation of higher education.
\end{abstract}

Keywords-Physical education (PE), higher education, teaching quality, analytic hierarchy process (AHP), grey system theory (GST)

\section{Introduction}

As a key aspect of quality education, physical education (PE) is a compulsory course in many colleges. The PE presents college students with a wide array of sports, making them more aware of the importance of physical fitness. Many colleges have strived to enhance the quality of PE teaching by reforming the PE courses [1-3].

With the rapid development of science, great changes have taken place in higher education. The models, philosophies, means and methods of higher education have been fully shaken up, raising higher requirements on educators [4-7]. Being a crucial link of higher education, PE teaching must also keep abreast of the times.

Many scholars have explored the problems in PE teaching from multiple angles and levels. For example, Xu and Yao [8] analyzed several factors that affect the PE teaching in colleges, and proposed relevant countermeasures. Kwon \& Block [9] investigated the training of PE teachers through adaptive PE e-learning. Lin et al. [10] discussed the innovative talent training model for PE majors. Forey \& Cheung [11] examined the benefits of explicit language teaching for PE classroom learning. Bailey et al. [12] analyzed the effect of the "three independence" PE reform, and provided detailed countermeasures. The "three independence" means students are independent to choose class time, teachers and sports. Sum et al. [13] demonstrated how continu- 
ing professional development affects the sports literacy and self-efficacy of PE teachers and the learning effect of students.

To sum up, the existing studies mainly focus on the theories and strategies of the $\mathrm{PE}$ teaching process, without clearly identifying the essential factors affecting the PE teaching quality or creating an evaluation system for PE teaching quality. Traditionally, several systemic analysis methods can make accurate reasoning under multiple influencing factors, namely, evidential reasoning (ER), genetic algorithms (GAs), and neural networks (NNs). However, the applicable scope of these methods is very limited, because they require complex mathematical models and a large sample size [1419].

Considering the factors affecting the quality of college PE teaching, this paper combines analytic hierarchy process (AHP) and grey system theory (GST) into a novel evaluation model for PE teaching quality in colleges [20-25].

The remainder of this paper is organized as follows: Section 2 analyzes the influencing factors of PE teaching in colleges today; Section 3 clarifies the hierarchy of teaching quality evaluation for college PE, and sets up a comprehensive index system; Sections 4 constructs an evaluation model for college PE teaching quality; Section 5 implements the AHP-based model in an actual case; Section 6 puts forward the conclusions.

\section{Analysis of Influencing Factors of Current College Students' Physical Education}

After consulted scholars engaged in PE teaching and higher education related fields, this paper applied questionnaire survey, expert interviews, literature reviews and other methods to explore and analyze the factors influencing the PE teaching in current colleges and universities, which are summarized as follows.

\subsection{Unsubstantial PE faculty}

In higher education, physical education is often less valued than other majors or disciplines. Especially in terms of faculty construction, the colleges' investment is usually insufficient, which has affected the faculty level and the teaching ability of college PE teachers. The quality of the faculty is the prerequisite and guarantee for improving the quality of physical education in colleges and universities. Without a first-level PE faculty, we can hardly cultivate PE students with top professional qualities. Therefore, optimizing college PE faculty level is crucial for guaranteeing the PE teaching quality of college students.

\subsection{Out-dated PE teaching mode}

Current PE teaching mode emphasizes on the traditional test-oriented teaching method, which adopts the doctrinaire knowledge teaching mode or mechanically combines PE major textbook content with a few body movements, while ignoring the 
quality education's requirements on PE teaching development, and resulting in that the teaching idea is out of touch with the pace of the times. At the same time, current PE teaching mode fails to effectively utilize advanced modern technologies, so there is still a big room to improve the teaching mode. Therefore, improving the existing teaching mode to make it keep up with the development pace of the times is one of the important means to improve the quality of college PE teaching.

\subsection{Insufficient ability to reform PE teaching}

By analyzing the survey results we can know that, current college PE teaching textbooks, syllabus, facilities and platforms haven't been updated for decades; and these changeless PE teaching infrastructures and groovelike teaching philosophy had deprived college PE teaching from vitalities and vividness. At the same time, although a few colleges have reformed their PE teaching textbooks, syllabus, facilities and platforms to some extent, but their measures are often aimless, making the PE teaching even more disorderly. Every new school leader brings a different idea to PE teaching, so the colleges do not have the ability to reform the PE teaching systematically. Therefore, improving the ability to reform PE teaching and systematically undertaking the teaching reform projects in a targeted manner is conductive to improving college PE teaching quality.

\subsection{Unreasonable PE curriculum planning}

The unreasonable PE curriculum planning has two meanings, one meaning is that the PE teaching content is not formulated according to the different learning stages, and the systematic and logical problems in the curriculum planning make the PE major knowledge fail to combine with the acceptability of the students; the other meaning is that the randomness of the PE teaching content fails to organically integrate with the PE teaching syllabus, and the progress of PE lessons can hardly be guaranteed, which is a very important factor affecting the quality of PE teaching.

\subsection{Disconnected PE teaching practice and theories}

It can be seen from the survey results that current college PE teaching mostly focuses on theoretical knowledge indoctrination. The teaching of basic knowledges such as PE concepts, skills, technics and terms is methodical and systematic. But if the theoretical knowledge fails to integrate with PE practice and the practice can't be verified by theoretical knowledge, these knowledges can only exist on the paper, which is neither conducive for students to digest the knowledge nor to improve their physical fitness. To this end, improving the integration of practice and theory in physical education is an important condition for ensuring the quality of PE teaching. 


\subsection{Low awareness of social service in PE teaching}

The goal of higher education is the quality education of college students, so the PE teaching should be conducted around this target as well. The ultimate goal of quality education is to serve the society; therefore, PE teaching should not be limited to the cultivation of PE knowledge and skills, it also should cultivate college students to serve the society from the aspects of sports culture, sports ethics, and sports concepts, etc., making them have the social service-oriented PE quality.

\subsection{Poor integration of industry-university-research in PE teaching}

In physical education, the so-called degree of industry-university-research cooperation refers to the degree of effective integration of the PE teaching, PE research and sports industry. As mentioned above, the ultimate goal of physical education is to serve the society, the sports industry is the joint point of PE teaching and the society, and the development of sports industry cannot be separated from the promotion of PE research. It can be seen that good interaction and integration of PE teaching, PE research and sports industry are the catalyst for promoting the quality of PE teaching.

\subsection{Improper settings of management organizations and systems of PE teaching}

At present, the setting of management organizations and systems of college PE teaching often has problems such as redundant organizations, unclear subjects of liability, cumbersome administrative affairs, unsystematic teaching management procedures, etc., resulting in inefficient PE teaching management organizations, and failing to providing effective support to PE teaching. However, such institutionalized and formalized settings of college PE teaching management organizations and systems are generally test-oriented, which is unsuitable for quality physical education. Therefore, to ensure good PE teaching quality, it's necessary to have properly-set PE teaching management organizations and systems.

\subsection{Imperfect PE teaching evaluation systems and methods}

If we want to find out the weak links in college PE teaching and take corresponding measures to improve them, we must have effective PE teaching evaluation systems and a set of methods. However, due to the influence of various factors in college $\mathrm{PE}$ teaching, the cultivation requirements and standards of college PE teaching are changing constantly with the progress of the times. Therefore, the PE teaching evaluation systems and methods should be improved and perfected continuously, so that they could better adapt to the PE teaching quality evaluation and analysis in contemporary colleges and universities, and thereby effectively improving the quality of college PE teaching. 


\section{$3 \quad$ AHP-Based College Student PE Teaching Quality Evaluation System}

\subsection{Principles for the selection of PE teaching quality evaluation indices}

After analyzing the influencing factors of college PE teaching, we'll need to select a few indices for the evaluation of the college PE teaching quality. Since the influencing factors of PE teaching are quite complex and varied, to ensure the evaluation effect of college PE teaching quality, there are several principles that should be followed for the selection of PE teaching quality evaluation indices.

Scientific and rational principle: The selected evaluation indices for the college PE teaching quality should have clear scientific significance, and can reasonably and accurately express the inherent meaning of PE teaching quality. Meanwhile, the evaluation indices should have clear logic and hierarchical relationships, so as to truly reflect the essential problems in the quality of PE teaching.

Authentic and objective principle: The selected evaluation indices for the college PE teaching quality should be based on the objective and actual situations in the college PE teaching process, and can authentically reflect the main influencing factors of the college PE teaching quality, thereby ensuring the authenticity and reliability of college PE teaching quality evaluation.

Typical principle: The evaluation of PE teaching quality is a complex decisionmaking process with multiple factors, if all influencing factors are taken as the evaluation indices for the evaluation of PE teaching quality, the decision-making process would be too complicated, and it's hard to obtain effective decision-making results. Therefore, in view of integrity and completeness, the selected evaluation indices should be typical and representative.

Quantifiable principle: Taking account of the diverse features of the evaluation indices of college PE teaching quality, to ensure good quantification effect of the evaluation results, when selecting the evaluation indices, it's necessary to make sure that the selected indices are highly convertible, so as to ensure the operability of the evaluation results of college PE teaching quality.

\subsection{Construction of PE teaching quality evaluation system}

Following the above principles and based the analysis of the influencing factors of college PE teaching quality, the authors constructed a novel college student PE teaching quality evaluation system from four aspects, namely: the ability to guarantee PE teaching infrastructures, the implementation effect of PE teaching; the ability to reform PE teaching; and the outcome of PE teaching.

The ability to guarantee PE teaching infrastructures, C1: This evaluation criterion is mainly to examine the college's ability to make sure of its basic conditions for the college PE teaching activities, and this is a prerequisite for guaranteeing the PE teaching quality of colleges and universities. Therefore, in the process of college PE teaching, we need to guarantee the PE teaching infrastructures in colleges and univer- 
sities, including the PE teaching input $\mathrm{C} 11$, PE faculty level C12, PE faulty construction $\mathrm{C} 13, \mathrm{PE}$ infrastructure construction $\mathrm{C} 14$, and $\mathrm{PE}$ administration rationality $\mathrm{C} 15$.

Implementation effect of PE teaching, C2: This evaluation criterion is mainly to examine the relevant factors affecting the PE teaching quality during the implementation of PE teaching, it's a main link for ensuring the quality of college PE teaching, therefore, the implementation effect of relevant links in PE teaching must be guaranteed, including the advancement of PE teaching mode $\mathrm{C} 21$, the rationality of $\mathrm{PE}$ teaching planning $\mathrm{C} 22$, the practicality of PE teaching $\mathrm{C} 23$, and the intelligent $\mathrm{PE}$ teaching methods $\mathrm{C} 24$.

Ability to reform PE teaching, C3: This evaluation criterion is mainly to examine the college's ability to reform and innovate PE teaching, it is an impetus for improving the quality of college PE teaching, therefore, we should actively carry out PE teaching reform works and enhance the ability to reform and innovate college PE teaching, including: PE teaching idea reform and innovation C31, ability to integrate $\mathrm{PE}$ teaching, PE research and sports industry $\mathrm{C} 32$, PE teaching content reform and innovation $\mathrm{C} 33$, and the ability to convert $\mathrm{PE}$ teaching reform results $\mathrm{C} 34$.

Outcome of PE teaching, C4: This evaluation criterion is mainly to examine the outcome of college PE teaching, which is the most direct reflection of the quality of college PE teaching, moreover, it can also reflect the implementation effects of the PE teaching reform links, therefore, it plays an irreplaceable role in the effective evaluation of PE teaching quality, including: the students' sport skill excellent rate $\mathrm{C} 41$, the students' PE research ability $\mathrm{C} 42$, the students' PE quality qualification rate $\mathrm{C} 43$, the students' PE culture awareness C44, the students' PE social service satisfaction C45, and the students' PE innovation ability C46.

\section{$4 \quad$ Evaluation model of College student PE Teaching Quality}

\subsection{AHP-based weight analysis}

After the AHP-based PE teaching quality evaluation system was constructed, we need to conduct weight analysis on the evaluation indices of the evaluation system. In order to construct judgment matrices for the evaluation indices under different hierarchies, and thereby obtaining the corresponding weight values, this paper adopted the 1-9 scaling method to assign values to the evaluation indices. The 1-9 scales characterize the relative importance of each pair of evaluation indices. If the importance degree of index ci relative to index cj is rij, then its specific meaning is listed below in Table 1. 
Table 1. Scale values and their meanings

\begin{tabular}{|c|l|}
\hline Scale value $\boldsymbol{r}_{i j}$ & \multicolumn{1}{|c|}{ Meanings } \\
\hline 1 & Index $c_{i}$ and index $c_{j}$ are equally important. \\
\hline 3 & Index $c_{i}$ is slightly more important than index $c_{j}$. \\
\hline 5 & Index $c_{i}$ is more important than index $c_{j}$. \\
\hline 7 & Index $c_{i}$ is much more important than index $c_{j}$. \\
\hline 9 & Index $c_{i}$ is extremely more important than index $c_{j}$. \\
\hline $2 / 4 / 6 / 8$ & The importance of index $c_{i}$ relative to index $c_{j}$ is between the adjacent two status. \\
\hline $1 / r_{i j}$ & The derivative of $r_{i j}$, it indicates the importance of index $c_{i}$ relative to index $c_{j}$. \\
\hline
\end{tabular}

Assuming there are $n$ evaluation indices in a certain hierarchy of the PE teaching quality evaluation system, by referring to expert opinions, scale values of 1-9 had been assigned to the evaluation indices, obtaining a judgment matrix $\mathrm{R}$ of the $\mathrm{n}$ evaluation indices as:

$$
\boldsymbol{R}=\left[\begin{array}{ccccc}
r_{11} & \cdots & r_{1 j} & \cdots & r_{1 n} \\
\vdots & \vdots & \vdots & \vdots & \vdots \\
r_{i 1} & \cdots & r_{i j} & \cdots & r_{i n} \\
\vdots & \vdots & \vdots & \vdots & \vdots \\
r_{n 1} & \cdots & r_{n j} & \cdots & r_{n n}
\end{array}\right]
$$

Based on the constructed judgment matrix $\mathrm{R}$, we can obtain the maximum eigenvalue $\lambda_{\max }(\boldsymbol{R})$ and the corresponding eigenvector $W(\boldsymbol{R})$ of the matrix, and thereby obtaining the consistency index $C I(\boldsymbol{R})$ of the judgment matrix $\mathrm{R}$ as:

$$
C I(\boldsymbol{R})=\frac{\lambda_{\max }(\boldsymbol{R})-n}{n-1}
$$

Using the number of the evaluation indices, namely the value of $\mathrm{n}$ to look up in the scale value table of the average random consistency index RI, and obtain the consistency ratio parameter $\mathrm{CR}(\mathrm{R})$ of the judgment matrix $\mathrm{R}$ :

$$
C R(\boldsymbol{R})=C I(\boldsymbol{R}) / R I
$$

If the consistency ratio parameter $\mathrm{CR}(\mathrm{R})$ of the judgment matrix $\mathrm{R}$ satisfies the following conditions, namely:

$$
C R(\boldsymbol{R})<0.1
$$

It indicates that the consistency of the judgment matrix $\mathrm{R}$ is appropriate. Otherwise, it indicates that the consistency of the judgment matrix $R$ does not meet the requirements, and the scale value rij needs to be re-assigned until the consistency of 
the judgment matrix $\mathrm{R}$ meets the requirements, and thereby obtaining the index weight sequence corresponding to the eigenvector $W(\boldsymbol{R})$, which is:

$$
W(\boldsymbol{R})=\left\{w_{1}, \cdots, w_{i}, \cdots w_{n}\right\}
$$

\subsection{GRA process}

From the construction process of the above-mentioned PE teaching quality evaluation system, it can be seen that the system has a hierarchical structure, and each hierarchy has several evaluation indices; and for each index in this hierarchy, its influence on the PE teaching quality is divided into several status. Suppose the PE teaching quality evaluation has $\mathrm{m}$ status, for the $\mathrm{j}$-th evaluation status of the $\mathrm{i}$-th evaluation index, the corresponding optimal status value is vij, the $\mathrm{n}$ evaluation indices form an optimal status sequence $\mathrm{Vj}$ with respect to the $\mathrm{j}$-th evaluation status:

$$
\boldsymbol{V}_{j}=\left\{v_{l j}, \cdots, v_{i j}, \cdots, v_{n j}\right\}
$$

If there is an evaluation object $\mathrm{P}$, for its $\mathrm{i}$-th evaluation index, the corresponding value is viP; assuming viP and vij are converted values with a unified scale, then the gray relational coefficient of the evaluation object $\mathrm{P}$ and the optimal status sequence $\mathrm{Vj}$ with respect to the $\mathrm{i}$-th evaluation index is:

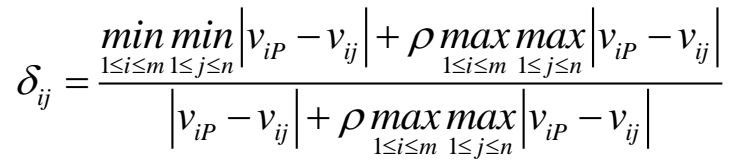

where, $\mathrm{i}=1,2, \cdots, \mathrm{n} ; \mathrm{j}=1,2, \cdots, \mathrm{m} ; \rho$ is the identification coefficient of GRA, $\rho \in$ $[0,1]$, generally, $\rho=0.5$.

\subsection{Model implementation}

The weight of the PE teaching quality evaluation indices under different hierarchical structures obtained based on AHP is $w_{i}$; and the gray relational coefficient between different PE teaching quality evaluation indices and evaluation status obtained based on GRA is $\delta_{i j}$, then the weighted gray relational $\phi_{j}$ between the evaluation object $\mathrm{P}$ and the optimal status sequence $\mathrm{Vj}$ is:

$$
\phi_{j}=w_{1} * \delta_{l j}+\cdots w_{i} * \delta_{i j}+\cdots+w_{n} * \delta_{n j}
$$

If there is:

$$
\phi_{k}=\max \left\{\phi_{1}, \cdots, \phi_{j}, \cdots, \phi_{m}\right\}, 1 \leq k, j \leq m
$$


It indicates that the evaluation object $\mathrm{P}$ has the highest correlation with the optimal status sequence $\mathrm{Vj}$. Therefore, the evaluation level of the PE teaching quality of evaluation object $\mathrm{P}$ is the $\mathrm{k}$-th level.

In summary, the implementation process of the PE teaching quality evaluation model based on AHP is shown as Figure 1 below.

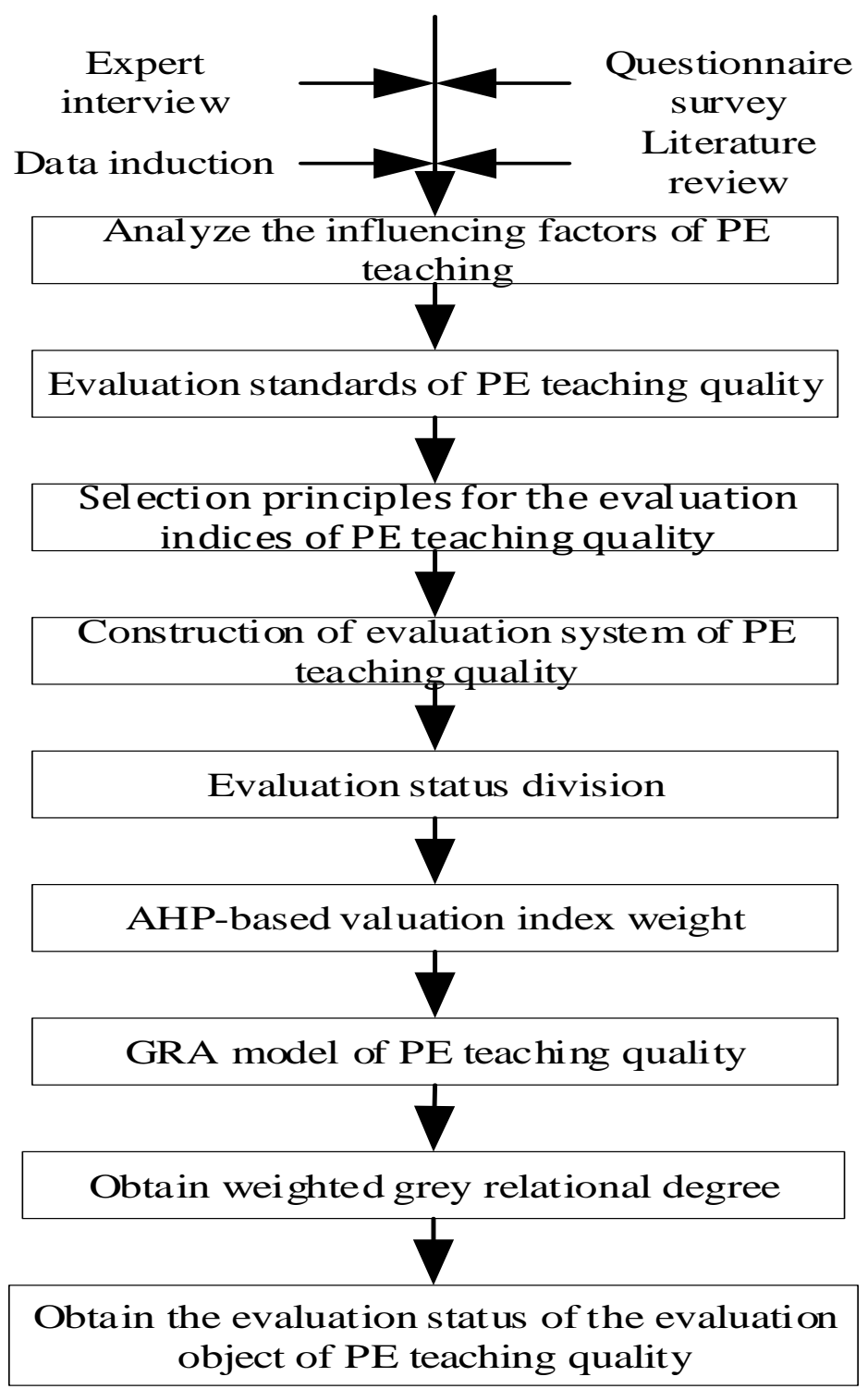

Fig. 1. Flow of the AHP-based PE teaching quality evaluation model 


\section{Case Study}

By referring to the opinions of relevant experts and combining with the actual situations in the analysis of the PE teaching quality, this paper divided the PE teaching quality evaluation into three modes, namely: high-level teaching quality LH, mediumlevel teaching quality LM, and low-level teaching quality LL, therefore, for each evaluation index of PE teaching quality, there are three status: high influence on PE teaching quality; medium influence on PE teaching quality; and low influence on PE teaching quality; and the corresponding value of the optimal status was 1.0, 0.8, and 0.6 , respectively. Through AHP, the weights of the indices under different hierarchical structures can be obtained, the specific results are shown in Table 2 below.

Table 2. AHP-based index weight

\begin{tabular}{|c|c|c|c|c|c|c|}
\hline Indices & \multicolumn{5}{|c|}{ Judgement matrices } & Weights \\
\hline$C_{1}, C_{2}, C_{3}, C_{4}$ & $\boldsymbol{R}_{C}=$ & $\begin{array}{l}1 \\
\frac{1}{2} \\
\frac{1}{3} \\
\frac{1}{2}\end{array}$ & $\begin{array}{l}2 \\
1 \\
\frac{1}{2} \\
1\end{array}$ & $\begin{array}{l}3 \\
2 \\
1\end{array}$ & 27 & $\begin{array}{l}0.424 \\
0.227 \\
0.122 \\
0.227\end{array}$ \\
\hline$c_{11}, c_{12}, c_{13}, c_{14}, c_{15}$ & $\boldsymbol{R}_{C_{l}}=$ & $\begin{array}{l}1 \\
4 \\
2 \\
3 \\
\frac{1}{2}\end{array}$ & $\begin{array}{l}\frac{1}{4} \\
1 \\
\frac{1}{2} \\
\frac{1}{2} \\
\frac{1}{7}\end{array}$ & $\begin{array}{l}3 \\
2 \\
\frac{1}{2}\end{array}$ & & $\begin{array}{l}0.096 \\
0.407 \\
0.171 \\
0.272 \\
0.054\end{array}$ \\
\hline$c_{21}, c_{22}, c_{23}, c_{24}$ & $\boldsymbol{R}_{C_{2}}=$ & $=\left[\begin{array}{l}1 \\
\frac{1}{2} \\
\frac{1}{3} \\
\frac{1}{5}\end{array}\right.$ & $\begin{array}{l}2 \\
1 \\
\frac{1}{2} \\
\frac{1}{3}\end{array}$ & 2 & & $\begin{array}{l}0.483 \\
0.272 \\
0.157 \\
0.088\end{array}$ \\
\hline
\end{tabular}




\begin{tabular}{|c|c|c|}
\hline$c_{31}, c_{32}, c_{33}, c_{34}$ & $\boldsymbol{R}_{C}$ & $y_{3}=$ \\
\hline$c_{41}, c_{42}, c_{43}, c_{44}, c_{45}, c_{46}$ & $\boldsymbol{R}_{C_{4}}=$ & 3 \\
\hline
\end{tabular}

Table 3. Weighted grey relational coefficients

\begin{tabular}{|c|c|c|c|c|}
\hline Indices & Converted values & LH & LM & LL \\
\hline$c_{11}$ & 0.825 & 0.054 & 0.096 & 0.047 \\
\hline$c_{12}$ & 0.940 & 0.345 & 0.256 & 0.157 \\
\hline$c_{13}$ & 0.660 & 0.065 & 0.108 & 0.145 \\
\hline$c_{14}$ & 0.850 & 0.166 & 0.241 & 0.126 \\
\hline$c_{15}$ & 0.735 & 0.024 & 0.045 & 0.034 \\
\hline$c_{21}$ & 0.620 & 0.161 & 0.248 & 0.437 \\
\hline$c_{22}$ & 0.800 & 0.133 & 0.272 & 0.133 \\
\hline$c_{23}$ & 0.685 & 0.059 & 0.098 & 0.108 \\
\hline$c_{24}$ & 0.800 & 0.043 & 0.088 & 0.043 \\
\hline$c_{31}$ & 0.750 & 0.202 & 0.337 & 0.253 \\
\hline$c_{32}$ & 0.500 & 0.071 & 0.097 & 0.152 \\
\hline$c_{33}$ & 0.625 & 0.162 & 0.238 & 0.368 \\
\hline$c_{34}$ & 0.500 & 0.026 & 0.036 & 0.056 \\
\hline$c_{41}$ & 0.875 & 0.040 & 0.048 & 0.027 \\
\hline$c_{42}$ & 0.680 & 0.056 & 0.094 & 0.108 \\
\hline$c_{43}$ & 0.800 & 0.122 & 0.261 & 0.122 \\
\hline$c_{44}$ & 0.700 & 0.062 & 0.108 & 0.108 \\
\hline$c_{45}$ & 0.950 & 0.078 & 0.054 & 0.033 \\
\hline$c_{46}$ & 0.780 & 0.108 & 0.218 & 0.120 \\
\hline & & & & \\
\hline
\end{tabular}

The obtained values of the evaluation object were converted to obtain values with uniform metrics. By applying the PE teaching quality GRA model proposed in this 
paper, the weighted gray relational coefficients between the evaluation indices and the evaluation status under different hierarchical structures were obtained. The specific results are shown in Table 3.

According to the PE teaching quality grey relational calculation model proposed in this paper, the obtained weighted grey relational degree between the evaluation object and the evaluation status is: $\phi_{L H}=0.529, \phi_{L M}=0.740$ and $\phi_{L L}=0.598$. It can be seen that the current PE teaching quality of the evaluation object belongs to the LM status.

\section{Conclusion}

The paper analyzed the influencing factors of the PE teaching quality in current colleges and universities, it gave relevant principles for the selection of evaluation indices of the college PE teaching quality, and constructed an evaluation system with hierarchical structures to assess the college PE teaching quality. Aiming at the constructed college PE teaching quality evaluation model, this paper combined AHP with GRA to propose an improved college PE teaching quality evaluation model, and used a practical example to verify it. The research results showed that the proposed method has good feasibility and operability, and it is an extension of the existing research results, which has a good reference significance for the analysis of complex systems with similar processing procedures.

\section{$7 \quad$ References}

[1] Triaca, L. M., Frio, G. S., \& França, M. T. A. (2019). A gender analysis of the impact of physical education on the mental health of Brazilian schoolchildren. SSM-population health, 8, 1-9. https://doi.org/10.1016/j.ssmph.2019.100419

[2] Packham, A., \& Street, B. (2019). The effects of physical education on student fitness, achievement, and behavior. Economics of Education Review, 72, 1-18. https://doi.org/10. $\underline{1016 / j . e c o n e d u r e v .2019 .04 .003}$

[3] Yang, Y., Meng, L. (2019). Physical education motion correction system based on virtual reality technology, International Journal of Emerging Technologies in Learning, 14(13), 105-116. https://doi.org/10.3991/ijet.v14i13.10710

[4] Thorburn, M. (2018). John Dewey, subject purposes and schools of tomorrow: A centennial reappraisal of the educational contribution of physical education. Learning, culture and social interaction, 19, 22-28. https://doi.org/10.1016/j.lcsi.2018.04.001.

[5] Cong, C. R., \& Wang, Z. X. (2019). The direction of P.E. curriculum reform in application-oriented colleges and universities. Journal of Kashi University, 40(6), 111-116. http://dx.doi.org/10.13933/j.cnki.2096-2134.2019.06.023

[6] Egan, C. A., Webster, C. A., Stewart, G. L., Weaver, R. G., Russ, L. B., Brian, A., \& Stodden, D. F. (2019). Case study of a health optimizing physical education-based comprehensive school physical activity program. Evaluation and program planning, 72, 106117. https://doi.org/10.1016/j.evalprogplan.2018.10.006 
[7] Wu, J. M., \& Zhang, C. X. (2019). A study on "Double-subject" teaching mode of sport statistics course. International Journal of Emerging Technologies in Learning, 14(18), 133144. https://doi.org/10.3991/ijet.v14i18.11386

[8] Xu, W. X., \& Yao, J. W. (2016). An Online Learning System Based on Panoramic Technology for Sport Psychology. International Journal of Emerging Technologies in Learning, 11(9), 20-24. https://doi.org/10.3991/ijet.v11i09.6115

[9] Kwon, E. H., \& Block, M. E. (2017). Implementing the adapted physical education Elearning program into physical education teacher education program. Research in developmental disabilities, 69, 18-29. https://doi.org/10.1016/j.ridd.2017.07.001

[10] Lin, Y., Pan, S. K., \& Shao, Y. (2001). On the development of students' teaching ability in the classroom of track and field. Journal of Tonghua Teachers College, 22(5), 87-89. https://doi.org/10.3969/j.issn.1008-7974.2001.05.029

[11] Forey, G., \& Cheung, L. M. E. (2019). The benefits of explicit teaching of language for curriculum learning in the physical education classroom. English for Specific Purposes, 54, 91-109. https://doi.org/10.1016/j.esp.2019.01.001

[12] Bailey, R., Armour, K., Kirk, D., Jess, M., Pickup, I., Sandford, R., \& Education, B. P. (2009). The educational benefits claimed for physical education and school sport: An academic review. Research papers in education, 24(1), 1-27. https://doi.org/10.1080/02671520 701809817

[13] Sum, K. W. R., Wallhead, T., Ha, S. C. A., \& Sit, H. P. C. (2018). Effects of physical education continuing professional development on teachers' physical literacy and self-efficacy and students' learning outcomes. International Journal of Educational Research, 88, 1-8. https://doi.org/10.1016/j.ijer.2018.01.001

[14] Sadeghi, A., Farhad, H., Moghaddam, A. M., \& Qazizadeh, M. J. (2018). Identification of accident-prone sections in roadways with incomplete and uncertain inspection-based information: a distributed hazard index based on evidential reasoning approach. Reliability Engineering \& System Safety, 178, 278-289. https://doi.org/10.1016/j.ress.2018.06.020

[15] Ganji, S. R. S., Rassafi, A. A., \& Xu, D. L. (2019). A double frontier DEA cross efficiency method aggregated by evidential reasoning approach for measuring road safety performance. Measurement, 136, 668-688. https://doi.org/10.1016/j.measurement.2018.12.098 0

[16] Korkmaz, Y., Boyac1, A., \& Tuncer, T. (2019). Turkish vowel classification based on acoustical and decompositional features optimized by Genetic Algorithm. Applied Acoustics, 154, 28-35. https://doi.org/10.1016/j.apacoust.2019.04.027

[17] Batle, J., Ciftja, O., Naseri, M., Ghoranneviss, M., Farouk, A., Elhoseny, M. (2017). Equilibrium and uniform charge distribution of a classical two-dimensional system of point charges with hard-wall confinement. Physica Scripta, 92(5), 055801. https://doi.org/10.10 88/1402-4896/aa6630

[18] Khwaja, A. S., Anpalagan, A., Naeem, M., \& Venkatesh, B. (2020). Joint bagged-boosted artificial neural networks: Using ensemble machine learning to improve short-term electricity load forecasting. Electric Power Systems Research, 179, 1-7. https://doi.org/10.1016 j.epsr.2019.106080

[19] Suntonsinsoungvon, E., \& Udpin, S. (2020). Exponential stability of discrete-time uncertain neural networks with multiple time-varying leakage delays. Mathematics and Computers in Simulation, 171, 233-245. https://doi.org/10.1016/j.matcom.2019.10.007

[20] Konstantinos, I., Georgios, T., \& Garyfalos, A. (2019). A Decision Support System methodology for selecting wind farm installation locations using AHP and TOPSIS: Case study in Eastern Macedonia and Thrace region, Greece. Energy Policy, 132, 232-246. https://doi. $\underline{\text { org/10.1016/j.enpol.2019.05.020 }}$ 
[21] Wolnowska, A. E., \& Konicki, W. (2019). Multi-criterial analysis of oversize cargo transport through the city, using the AHP method. Transportation Research Procedia, 39, 614-623. https://doi.org/10.1016/j.trpro.2019.06.063

[22] Gürcan, Ö. F., Yazıcı, I., Beyca, Ö. F., Arslan, Ç, Y., \& Eldemire, F. (2016). Third party logistics (3PL) provider selection with AHP application. Procedia-Social and Behavioral Sciences, 235, 226-234. https://doi.org/10.1016/j.sbspro.2016.11.018

[23] Balsara, S., Jain, P. K., \& Ramesh, A. (2019). An integrated approach using AHP and DEMATEL for evaluating climate change mitigation strategies of the Indian cement manufacturing industry. Environmental pollution, 252, 863-878. https://doi.org/10.1016/j.env pol.2019.05.059

[24] Das, R., Ball, A. K., \& Roy, S. S. (2018). Optimization of E-jet Based Micro Manufacturing Process Using Grey Relation Analysis. Materials Today: Proceedings, 5(1), 200-206. https://doi.org/10.1016/j.matpr.2017.11.072

[25] Lee, P. T. W., Lin, C. W., \& Shin, S. H. (2012). A comparative study on financial positions of shipping companies in Taiwan and Korea using entropy and grey relation analysis. Expert Systems with Applications, 39(5), 5649-5657. https://doi.org/10.1016/j.eswa.2011. $\underline{11.052}$

\section{Author}

Ke Han was born in Jinan, Shandong, China in 1992. He received the master's degree from Beijing Sport University, China. Now works at School of Physical education department, Harbin institute of Technology, Weihai 264209, China His research interest includes physical education, movement training and Sports technology research. hankejiayou@163.com.

Article submitted 2020-01-22. Resubmitted 2020-03-05. Final acceptance 2020-03-06. Final version published as submitted by the authors. 